Pacific Journal of Mathematics

ON EQUIVALENT CATEGORY BASES 


\title{
ON EQUIVALENT CATEGORY BASES
}

\author{
JOHN. C. MORGAN II
}

\begin{abstract}
Any category base in which every region contains a minimal region is equivalent to a topology.
\end{abstract}

Utilizing the notion of a category base, which is a generalization of the notion of a topology, the author has developed a general theory of point sets within which many of the analogies between Lebesgue measure and Baire category have been unified (cf. [3]-[9]). In view of the "equivalence" between the Lebesgue measurable sets and the sets having the Baire property relative to the density topology (cf. [10, Chapter 22]), the question arises whether every category base is equivalent to some topology. We do not know the answer to this question. However, we shall show in this article that for a certain class of category bases, including all finite category bases, there do exist equivalent topologies.

After stating pertinent facts in $\$ 1$, we define in $\$ 2$ a basic topology which is associated with a given category base. In $\$ 3$ we determine this basic topology in several examples and see that it is not in general equivalent to the category base from which it arises. As we show in $\S 4$, however, any category base in which every region contains a minimal region is equivalent to its basic topology.

1. Preliminaries. In this section we recall relevant definitions and theorems which were established in [4].

Definition. A pair $(X, \mathcal{C})$, where $e$ is a family of subsets of a

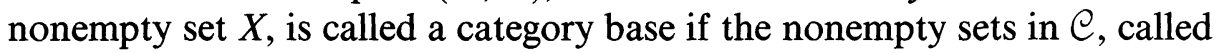
regions, satisfy the following axioms:

1. Every point of $X$ belongs to some region; i.e. $X=\cup \mathcal{C}$.

2. Let $A$ be a region and let $\mathscr{D}$ be any nonempty family of disjoint regions which has power less than the power of $\mathcal{C}$.

(a) If $A \cap(\cup \mathscr{D})$ contains a region then there is a region $D \in \mathscr{D}$ such that $A \cap D$ contains a region.

(b) If $A \cap(\cup \mathscr{D})$ contains no region then there is a region $B \subset A$ which is disjoint from every region in $\mathscr{D}$.

It is readily seen that every topology is a category base.

With respect to a given category base we have the following generalization of the topological notions of nowhere dense sets, sets of the first category, and sets of the second category. 
DEFINITION. A set $S$ is singular if every region contains a subregion which is disjoint from $S$. A countable union of singular sets is called a meager set. A set which is not a meager set is called an abundant set.

Notation. The family of all sets which are meager with respect to $(X, \mathcal{C})$ is denoted by $\mathfrak{M}(\mathcal{C})$.

THEOREM 1.1. The intersection of any two regions either contains a region or it is a singular set.

THEOREM 1.2. The family of all singular sets forms an ideal and the family of all meager sets forms a o-ideal.

Definition. A set $S$ is meager (abundant) in a region $A$ if $S \cap A$ is meager (abundant). A set is abundant everywhere in a region $A$ if it is abundant in every subregion of $A$.

The appropriate generalization of the topological notion of a set being of the first category at a point is

Definition. A set $S$ is locally meager at a point $x \in X$ if in every region $A$ containing $x$ there is a subregion $B$ containing $x$ such that $S \cap B$ is a meager set. Otherwise, $S$ is said to be locally abundant at the point $x$.

THEOREM 1.3. The set of all points of a set $S$ at which $S$ is locally meager is a meager set.

Definition. A set $S$ has the Baire property if every region $A$ has a subregion $B$ in which either $S$ or $X-S$ is a meager set. Restated, $S$ has the Baire property if there is no region in which both $S$ and its complement are abundant everywhere.

NotATion. The family of all sets which have the Baire property with respect to $(X, \mathcal{C})$ is denoted by $\mathfrak{B}(\mathcal{C})$.

THEOREM 1.4. The sets which have the Baire property form a $\sigma$-field which contains all regions and all meager sets.

Definition. Two category bases $(X, \mathcal{C})$ and $(X, \mathscr{D})$ are called equivalent if $\mathfrak{M}(\mathcal{C})=\mathfrak{M}(\mathscr{D})$ and $\mathfrak{B}(\mathcal{C})=\mathfrak{B}(\mathscr{D})$. 
2. The basic topology. We assume throughout this section a given category base $(X, \mathcal{e})$.

Definition. The set of all points of $X$ at which a set $S$ is locally abundant will be called the derived set of $S$ and denoted by $D(S)$. That is, a point $x$ belongs to $D(S)$ if and only if there is a region $A$ containing $x$ such that for every subregion $B$ of $A$ which contains $x$, the set $S \cap B$ is an abundant set.

One can readily verify the following properties of the derived set operation (cf. $[2, \S 10])$ :

(1) If $S$ is meager then $D(S)=\varnothing$.

(2) If $S \subset T$ then $D(S) \subset D(T)$.

(3) $D(S \cup T)=D(S) \cup D(T)$.

(4) For any index set $I, \cup_{\alpha \in I} D\left(S_{\alpha}\right) \subset D\left(\cup_{\alpha \in I} S_{\alpha}\right)$.

(5) For any index set $I, D\left(\cap_{\alpha \in I} S_{\alpha}\right) \subset \cap_{\alpha \in I} D\left(S_{\alpha}\right)$.

(6) $D(S)-D(T) \subset D(S-T)$.

From Theorem 1.3 we have

(7) $S-D(S)$ is a meager set.

We then obtain from the decomposition

$$
S=[S \cap D(S)] \cup[S-D(S)]
$$

(8) $S$ is meager if and only if $D(S)=\varnothing$.

Applying $D$ to the above decomposition yields

$$
\begin{aligned}
D(S) & =D[S \cap D(S)] \cup D[S-D(S)] \\
& =D[S \cap D(S)] \subset D(S) \cap D(D(S)) \subset D(D(S)) .
\end{aligned}
$$

Hence

(9) $D(S) \subset D(D(S))$.

Definition. A set $S$ is called closed if $D(S) \subset S$. A set $S$ is called homogeneous if $S \subset D(S)$.

THEOREM 2.1. The family $\mathcal{F}$ of all closed sets has the properties:

(i) $\varnothing, X$ belong to $\mathscr{F}$.

(ii) Every meager set belongs to $\mathscr{F}$.

(iii) $\mathscr{F}$ is closed under finite unions.

(iv) $\mathscr{F}$ is closed under arbitrary intersections.

Definition. The complement of a closed set is called an open set. 
THEOREM 2.2. The family $\mathrm{C}^{*}$ of all open sets is a topology.

We shall call the topology $\left(X, \mathrm{e}^{*}\right)$ the basic topology for the category base $(X, \mathcal{C})$. In the case that $(X, \mathcal{C})$ is a $T_{1}$-topology, the basic topology $\left(X, \bigodot^{*}\right)$ was investigated by Hashimoto [1].

3. Examples. In this section we determine the basic topology $\left(X, \mathcal{C}^{*}\right)$ for several category bases $(X, \mathcal{C})$.

EXAMPLE 3.1. If $(X, \mathcal{C})$ is a Baire topology (i.e. every nonempty open set is of the second category) which satisfies CCC then $\left(X, \mathrm{e}^{*}\right)$ is equivalent to $(X, \mathrm{e})$. The topology $\left(X, \mathrm{C}^{*}\right)$, in fact, consists of all sets of the form $G-I$, where $G$ is an open set and $I$ is a set of the first category with respect to $(X, \mathcal{e})$. In the case that $(X, \mathcal{C})$ is the usual topology on the real line, $(X, \mathcal{C})$ has a countable topological base for the open sets, but the topology $\left(X, e^{*}\right)$ does not even have a countable pseudo-base.

EXAMPLE 3.2. Let $(X, \mathcal{C})$ be the cofinite topology on the set $X$ of all natural numbers. The topology $\left(X, \mathrm{e}^{*}\right)$ is the discrete topology and is not equivalent to $(X, \mathcal{C})$, although $\mathfrak{B}(\mathcal{C})=\mathfrak{B}\left(\mathrm{C}^{*}\right)$. Note that $(X, \mathcal{C})$ is not a Baire topology, while $\left(X, \bigodot^{*}\right)$ is a Baire topology.

EXAMPLE 3.3. Let $(X, \overline{\mathbb{Q}}, \bar{\mu})$ be the completion of a continuous, $\sigma$-finite measure space $(X, \mathscr{Q}, \mu)$ and let $\mathcal{C}$ be the family of all sets in $\mathbb{Q}$ of positive measure. The meager sets coincide with the sets of $\bar{\mu}$-measure zero and the sets having the Baire property with respect to $(X, \mathcal{C})$ are the $\bar{\mu}$-measurable sets. It can be shown that, for every set $S$ of positive $\bar{\mu}$-outer measure, $D(S)=X$. This implies the topology $\left(X, \mathrm{e}^{*}\right)$ consists only of $\varnothing$, $X$, and every set whose complement has $\bar{\mu}$-measure zero. Hence, $\left(X, \mathrm{C}^{*}\right)$ is not in general equivalent to $(X, \mathcal{e})$.

EXAmple 3.4. Let $X$ denote $n$-dimensional Euclidean space and let $e$ be the family of all closed sets which are of positive Lebesgue measure in every neighborhood of each of their points. The meager sets coincide with the sets of Lebesgue measure zero and the sets having the Baire property with respect to $(X, \mathcal{C})$ coincide with the Lebesgue measurable sets. It can be shown that the topology ( $X, e^{*}$ ) consists of all sets of the form $G-N$, where $G$ is an open set in $X$ and $N$ is a set of Lebesgue measure zero. Consequently, each nowhere dense set in $X$, as well as each set of Lebesgue measure zero, is nowhere dense with respect to $\left(X, \mathrm{e}^{*}\right)$. In view of the fact that $X$ can be decomposed into an $\mathscr{F}_{\sigma}$-set of the first category in $X$ and a $\mathcal{G}_{\delta}$-set of Lebesgue measure zero, every subset of $X$ is of the first category with respect to $\left(X, \mathrm{C}^{*}\right)$. Hence, $\left(X, \mathrm{\complement}^{*}\right)$ is not a Baire topology 
and $\mathfrak{B}\left(\mathrm{C}^{*}\right)$ consists of all subsets of $X$, so $(X, \mathcal{C})$ and $\left(X, \complement^{*}\right)$ are not equivalent. A comparison of this example with the preceding one reveals the fact that equivalent category bases need not have equivalent basic topologies.

EXAMPLE 3.5. Let $X$ be a complete separable metric space with no isolated points and let $\mathcal{C}$ be the family of all perfect sets in $X$. The sets having the Baire property with respect to $(X, \mathcal{C})$ coincide with a classification of sets investigated by Marczewski (cf. [7, Example 3], [11]). The topology $\left(X, \mathrm{C}^{*}\right)$ consists of all sets of the form $G-I$, where $G$ is an open set in $X$ and $I$ is a Marczewski singular set. Accordingly, every nowhere dense set in $X$ is nowhere dense with respect to $\left(X, \mathrm{e}^{*}\right)$. Because every nowhere dense perfect set in $X$ contains a set $S \notin \mathfrak{B}(\mathcal{C})$, while $S \in \mathfrak{B}\left(\mathfrak{C}^{*}\right)$, we have $\mathfrak{B}(\mathcal{C}) \neq \mathfrak{B}\left(\mathfrak{C}^{*}\right)$. Hence, $(X, \mathcal{C})$ and $\left(X, \mathfrak{C}^{*}\right)$ are not equivalent. Assuming the continuum hypothesis (or the weaker hypothesis that there is no weakly inaccessible cardinal number less than or equal to $2^{\aleph_{0}}$ ), there exists a set which does not have the Baire property with respect to $\left(X, \mathrm{e}^{*}\right)$ (see $[4$, Theorem 16]).

\section{Category bases in which every region contains a minimal region.}

Definition. A region is a minimal region if it has no proper subregions.

We assume throughout this section that $(X, \mathcal{C})$ is a category base in which every region contains a minimal region. We denote the family of all minimal regions by $\mathscr{B}$ and set $Y=\cup \mathscr{B}$.

THEOREM 4.1. If $A$ is any region and $B$ is a minimal region then either $A \cap B=\varnothing$ or $B \subset A$.

Proof. According to Theorem 1.1, either $A \cap B$ is singular or $A \cap B$ contains a region.

If $A \cap B$ is singular then there is a region $C \subset B$ such that $A \cap C=$ $\varnothing$. From the minimality of $B$, we have $C=B$. Hence $A \cap B=\varnothing$.

If $A \cap B$ contains a region $C$ then, by the minimality of $B$, we have $C=B$. From the inclusions $C \subset A \cap B \subset A$, we obtain $B \subset A$.

THEOREM 4.2. Any two distinct minimal regions are disjoint.

THEOREM 4.3. $A$ set $S$ is singular if and only if $S \subset X-Y$. 
Proof. If $S \subset X-Y$ then $S$ is obviously singular.

Suppose $S$ is a set and $S \cap Y \neq \varnothing$. Then there is a region $B \in \mathscr{B}$ such that the set $S \cap B \neq \varnothing$. Due to the minimality of $B$, no subregion of $B$ is disjoint from $S$. Accordingly, $S$ is not a singular set. Therefore, if $S$ is a singular set then $S \cap Y=\varnothing$ and consequently $S \subset X-Y$.

From this theorem we derive the following three.

THEOREM 4.4. $A$ set $S$ is meager if and only if $S \subset X-Y$.

THEOREM 4.5. Every meager set is singular.

THEOREM 4.6. $(X, \mathcal{C})$ is a Baire base; i.e. every region is an abundant set.

THEOREM 4.7. If $S$ has the Baire property, $B$ is a minimal region, and $S \cap B \neq \varnothing$ then $B \subset S$.

Proof. According to Theorem 4.4, $S \cap B$ is an abundant set. By the minimality of $B$, the set $S$ is abundant everywhere in $B$. Since $S$ has the Baire property, $(X-S) \cap B$ must be a meager set. From Theorem 4.4, we have

$$
(X-S) \cap B \subset X-Y \subset X-B .
$$

But this impllies $(X-S) \cap B=\varnothing$. Therefore, $B \subset S$.

THEOREM 4.8. A set $S$ has the Baire property if and only if it is representable in the form

$$
S=E \cup P
$$

where $E$ is a union of minimal regions and $P$ is a singular set.

Proof. Suppose $S$ has the Baire property. Let $\mathscr{B}^{\prime}$ be the family of all minimal regions $B \in \mathscr{B}$ such that $B \subset S$, let $E=\cup \mathscr{B}^{\prime}$, and let $P=S-$ $E$. By virtue of Theorem 4.7, we have $S \cap B=\varnothing$ for every region $B \in \mathscr{B}-\Re^{\prime}$. Hence $P \cap B=\varnothing$ for every $B \in \mathscr{B}$. According to Theorem 4.3, $P$ is a singular set. We thus obtain a representation of the desired form.

Conversely, suppose $S$ has the form (*). Let $A$ be any region. If $A \cap E=\varnothing$ then $A \cap S=A \cap P$ is a meager set. If $A \cap E \neq \varnothing$ then, by Theorem 4.1, there is a region $B \in \Re$ such that $B \subset A \cap E$. This implies $B \cap(X-S)=\varnothing$ is a meager set. We conclude $S$ has the Baire property.

We turn now to investigate the basic topology $\left(X, \bigodot^{*}\right)$ associated with $(X, \mathcal{e})$. 
THEOREM 4.9. $X$ is homogeneous.

Proof. Suppose $x \in X$ and let $A$ be a region containing $x$. If $B$ is any subregion of $A$ which contains $x$ then, by Theorem 4.6, the set $X \cap B=B$ is an abundant set. Hence $x \in D(X)$, so $X \subset D(X)$.

THEOREM 4.10. Every nonempty open set is an abundant set.

Proof. Let $S$ be a meager set. From Theorem 4.9, we have $D(X)=X$. Using properties (1) and (6) of the derived set operation, we obtain

$$
X=D(X)=D(X)-D(S) \subset D(X-S) .
$$

If $S$ is also an open set then $X-S$ is closed and accordingly $D(X-S)$ $\subset X-S$. But $X \subset X-S$ implies $S=\varnothing$.

THEOREM 4.11. If $S$ is closed then $S$ has the Baire property with respect to $(X, \mathcal{C})$.

Proof. Suppose $S$ does not have the Baire property. Then there is a region $B \in \mathscr{B}$ such that $S \cap B$ and $(X-S) \cap B$ are abundant sets. Since $B \subset D(S)$ and $(X-S) \cap B \neq \varnothing$, we cannot have $D(S) \subset S$. Hence $S$ is not closed.

THEOREM 4.12. A necessary and sufficient condition that a set $S$ representable in the form $(*)$ be a closed set is that for every point $x \in$ $(X-Y)-P$, every region $A$ containing $x$ has a subregion $B$ containing $x$ such that $B \cap E=\varnothing$.

Proof. Assume first of all that the condition holds. Suppose $x \notin S$ and let $A$ be any region containing $x$.

If $x \in Y$ then it follows from Theorem 4.2 and the fact that $x \notin E$ that there exists a region $B \in \mathscr{B}$ such that $x \in B$ and $B \cap E=\varnothing$. Then $A \cap B \neq \varnothing$. By Theorem 4.1, we have $B \subset A, x \in B$, and $B \cap S=B \cap P$ is a meager set.

On the other hand, if $x \notin Y$ then $x \in(X-Y)-P$ and, using the assumed condition, there is a region $B \subset A$ such that $x \in B$ and $B \cap S=$ $B \cap P$ is a meager set.

In either case we obtain $x \notin D(S)$. This implies $D(S) \subset S$. Thus, $S$ is a closed set.

Assume next that the condition does not hold. Then there exists a point $x \in(X-Y)-P$, whence $x \in X-S$, and there exists a region $A$ containing $x$ such that for every region $B \subset A$ with $x \in B$ we have 
$B \cap E \neq \varnothing$. Now, $B \cap E \neq \varnothing$ implies there is a minimal region $C \subset E$ such that $B \cap C \neq \varnothing$. By Theorem 4.1, we have $C \subset B \cap E \subset B \cap S$. According to Theorem 4.6, $C$ is an abundant set. Thus, $B \cap S$ is an abundant set for every region $B \subset A$ with $x \in B$. This means $x \in D(S)$. But, because $x \notin S$, the set $S$ is not a closed set.

THEOREM 4.13. Any union of minimal regions is an open set.

Proof. The condition in Theorem 4.12 is vacuously satisfied whenever $P=X-Y$. Hence, the complement of any union of minimal regions is a closed set.

THEOREM 4.14. Every open subset of $Y$ is a union of minimal regions.

Proof. Let $S$ be an open subset of $Y$. From Theorems 4.11 and 4.8 it follows that $S=E \cup P$, where $E$ is a union of minimal regions and $P$ is a singular set. In view of Theorem 4.3, we have $P=\varnothing$.

THEOREM 4.15. Every minimal region is a minimal nonempty open set.

Proof. If $B$ is a minimal region then, according to Theorem $4.13, B$ is a nonempty open set. Moreover, if $H$ is any nonempty open subset of $B$ then Theorem 4.14 implies $H=B$.

THEOREM 4.16. The nowhere dense sets coincide with the singular sets.

Proof. In view of Theorem 4.3, we need only show a set $S$ is nowhere dense if and only if $S \subset X-Y$.

To show any subset of $X-Y$ is nowhere dense it suffices to prove $X-Y$ itself is nowhere dense. Let $G$ be any nonempty open set. From Theorems 1.4 and 4.11 we know $G$ has the Baire property with respect to ( $X, \mathcal{C}$ ). According to Theorem 4.8, $G=E \cup P$, where $E$ is a union of sets in $\Re$ and $P$ is a singular set. In view of Theorem 4.10, we have $E \neq \varnothing$. Let $H$ be a region in $\mathscr{B}$ such that $H \subset E$. By Theorem 4.13, $H$ is a nonempty open set. We have $H \subset G$ and $H \cap(X-Y)=\varnothing$. Therefore, $X-Y$ is a nowhere dense set.

Assume now that $S$ is not a subset of $X-Y$. Then there is a region $B \in \mathscr{B}$ such that $S \cap B \neq \varnothing$. The set $B$ is a nonempty open set which, according to Theorem 4.15, contains no nonempty open set disjoint from $S$. Therefore, $S$ is not a nowhere dense set.

From this theorem we easily derive the following three theorems. 
THEOREM 4.17. The sets of the first category coincide with the meager sets.

THEOREM 4.18. Every set of the first category is nowhere dense.

Theorem 4.19. ( $\left.X, \mathrm{C}^{*}\right)$ is a Baire topology.

We arrive finally at our main result.

Equivalence Theorem. If $(X, \mathcal{C})$ is a category base in which every region contains a minimal region then $(X, \mathcal{C})$ is equivalent to the topology $\left(X, e^{*}\right)$.

Proof. By Theorem 4.17, we have $\mathfrak{M}(\bigodot)=\mathfrak{M}\left(\bigodot^{*}\right)$.

If $S \in \mathfrak{B}(\mathcal{C})$ then we see from Theorems $4.8,4.13$, and 4.16 that $S$ is the union of an open set and a nowhere dense set, so $S \in \mathfrak{B}\left(\mathrm{C}^{*}\right)$.

Suppose, on the other hand, that $S \notin \mathfrak{B}(\mathcal{C})$. Then there exists a minimal region $B$ in which both $S$ and $X-S$ are abundant everywhere. From Theorems 4.15 and 4.17 it follows that $B$ is a nonempty open set in which both $S$ and $X-S$ are everywhere of the second category. Hence $S \notin \mathfrak{B}\left(\mathfrak{C}^{*}\right)$.

We thus have $\mathfrak{B}\left(C^{\prime}\right)=\mathfrak{B}\left(\bigodot^{*}\right)$.

COROLlaRy. Every finite category base is equivalent to a topology.

\section{REFERENCES}

1. H. Hashimoto, On the *topology and its application, Fund. Math., 91 (1976), 5-10.

2. K. Kuratowski, Topology, Volume 1, Academic Press, New York, 1966.

3. J. C. Morgan II, Infinite games and singular sets, Colloq. Math., 29 (1974), 7-17.

4. __ Baire category from an abstract viewpoint, Fund. Math., 94 (1977), 13-23.

5. __ On translation invariant families of sets, Colloq. Math., 34 (1975), 63-68.

$6 . \quad$ _ On zero-one laws, Proc. Amer. Math. Soc., 62 (1977), 353-358.

$7 . \quad$ _ The absolute Baire property, Pacific J. Math., 65 (1976), 421-436.

8. _ On the absolute Baire property, ibid. 78 (1978), 415-431.

$9 . \quad$ On product bases, ibid. 99 (1982), 105-126.

10. J. C. Oxtoby, Measure and Category, Springer, New York, 1971.

11. E. Szpilrajn (Marczewski), Sur une classe de fonctions de M. Sierpiński et la classe correspondante d'ensembles, Fund. Math., 24 (1935), 17-34.

Received August 26, 1981 and in revised form October 19, 1981.

California State Polytechnic University

POMONA, CA 91768 



\section{PACIFIC JOURNAL OF MATHEMATICS}

EDITORS

Donald BABBITT (Managing Editor)

University of California

Los Angeles, CA 90024

Hugo Rossi

University of Utah

Salt Lake City, UT 84112

C. C. Moore and Arthur Ogus

University of California

Berkeley, CA 94720
J. DugunduI

Department of Mathematics

University of Southern California

Los Angeles, CA 90089-1113

R. FinN and H. SAmelson

Stanford University

Stanford, CA 94305

\section{ASSOCIATE EDITORS}
R. ARENS
E. F. BECKENBACH
B. H. NEUMANN
F. WolF
K. YoshidA (1906-1982)

\section{SUPPORTING INSTITUTIONS}

UNIVERSITY OF ARIZONA

UNIVERSITY OF BRITISH COLUMBIA

CALIFORNIA INSTITUTE OF TECHNOLOGY

UNIVERSITY OF CALIFORNIA

MONTANA STATE UNIVERSITY

UNIVERSITY OF NEVADA, RENO

NEW MEXICO STATE UNIVERSITY

OREGON STATE UNIVERSITY
UNIVERSITY OF OREGON

UNIVERSITY OF SOUTHERN CALIFORNIA

STANFORD UNIVERSITY

UNIVERSITY OF HAWAII

UNIVERSITY OF TOKYO

UNIVERSITY OF UTAH

WASHINGTON STATE UNIVERSITY

UNIVERSITY OF WASHINGTON 


\section{Pacific Journal of Mathematics}

Vol. 105, No. 1 September, 1983

Kenneth F. Andersen, On the transformation of Fourier coefficients of

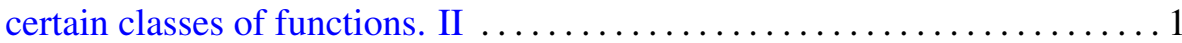

Gavin Brown, Irving Leonard Glicksberg and Edwin Hewitt, Indicator functions with large Fourier transforms $\ldots \ldots \ldots \ldots \ldots \ldots \ldots \ldots \ldots \ldots$

Shih-Sen Chang, Some random fixed point theorems for continuous random

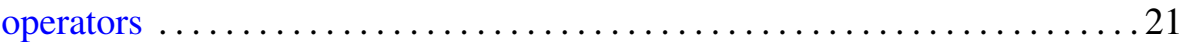

K. C. Chattopadhyay and Olav Njstad, Quasiregular nearness spaces and

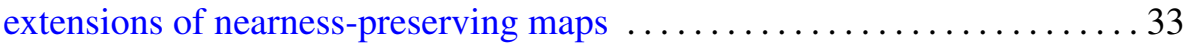

Thomas W. Cusick, The two-dimensional Diophantine approximation

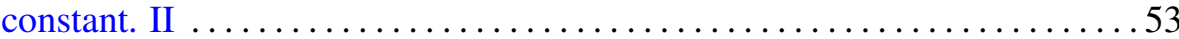

Eric Karel van Douwen and Jan van Mill, Spaces without remote points . . .669 Hector O. Fattorini, Convergence and approximation theorems for

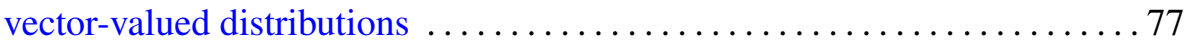

John J. F. Fournier and Louis Pigno, Analytic and arithmetic properties of

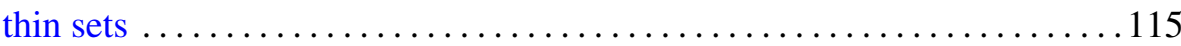

David Goss, On a new type of $L$-function for algebraic curves over finite

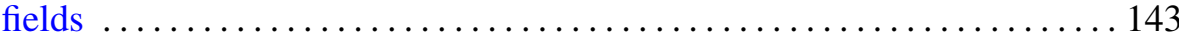

Douglas Austin Hensley, Lattice vertex polytopes with interior lattice

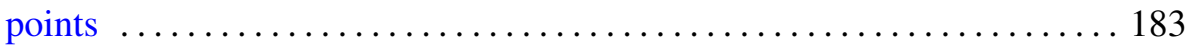

J. K. Kohli, Monotone extensions of mappings and their applications ...... 193

John C. Morgan, II, On equivalent category bases . . . . . . . . . . . . 207

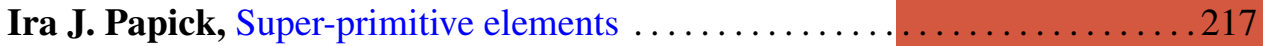

José Luis Rubio de Francia and José Luis Torrea, Vector extensions of

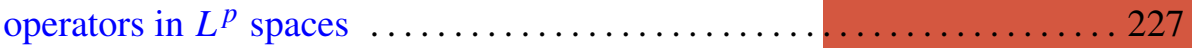

Mark Phillip Thomas, Closed ideals of $l^{1}\left(\omega_{n}\right)$ when $\left\{\omega_{n}\right\}$ is star-shaped $\ldots 237$ 